\title{
A computer model of sediment dynamics and radionuclide dispersion in a macrotidal estuary
}

\author{
P. Gleizon, A.G. Punt and M.G. Lyons ${ }^{1}$ \\ Westlakes Research Institute, The Princess Royal Building, \\ Westlakes Science and Technology Park, Moor Row, Cumbria CA24 3LN, U.K. \\ ${ }^{1}$ Westinghouse/BNFL Fuel Business, Springfields, Salwick, Preston PR4 OXJ, U.K.
}

\begin{abstract}
In macrotidal, partially mixed estuaries, tidal pumping can become a dominant mechanism that progressively accumulates sediment in the upper part of the estuary. This mechanism is also reinforced by different mixing behaviour during flood and ebb, due to significantly different stratification patterns. The flux and retention of particle reactive radionuclides within an estuary is complicated by their adsorption onto suspended silts and clays that may then accumulate in an estuary due to these tidal pumping processes. Predicting the accumulation of radionuclides in an estuary is further complicated by radioactive decay processes where daughter products may have distinctly different geochemical properties to those of the parent. The VERSE model has been recently developed in order to replicate the sediment transport and the advection-dispersion of dissolved and particle bound radionuclides with up to nine daughter products. The model is two-dimensional laterally averaged and uses finite difference schemes on a fixed grid. It contains four modules that compute the hydrodynamics, the cohesive sediment dynamics, and the advectiondispersion of conservative and non-conservative contaminants. VERSE has been developed to provide industry and regulatory authorities with a tool that can predict the transport of sediment and the dispersion of contaminants in estuaries, under various hydrodynamic conditions. This paper provides an overview of the model structure and results are compared to field data collected in a macrotidal estuary during several measurement campaigns.
\end{abstract}

\section{INTRODUCTION}

Estuaries are complex environmental systems. Extreme flow conditions such as high tidal range and high or low river run-off, influence whether sediment is retained or flushed from an estuary. Tidal pumping has been considered as one of the key processes that explain the landward transport of sediment ([1], [2]). In partially mixed estuaries, tidal pumping is further complicated by vertical stratification that can significantly influence the dispersion of particle bound contaminants. Depth-averaged models fail to replicate these features, and in stratified systems models need to be able to resolve variations with depth if they are to be used as decision support tools.

\section{MODEL. DESIGN}

\subsection{Assumptions}

VERSE is designed for relatively narrow macrotidal estuaries where transverse circulation is assumed to have minimal impact on the along-channel dispersion. In order to model the transport of both dissolved and particulate nuclides, the model includes the simulation of the hydrodynamics and the erosiondeposition and subsequent advection-dispersion of fine-grained sediment. In partially mixed estuaries saline stratification and the vertical gradient of suspended particulate matter (SPM) concentration therefore need to be replicated by the model.

The modelling domain consists of a fixed grid, orientated in the along-channel and the vertical directions. The grid resolution is 250 meters along the axis of the estuary, and 0.3 meters in the vertical 
direction. The fine vertical grid resolution is needed to accurately represent water depths towards the head of the estuary when depths as small as 0.6 meters can occur.

The complexity of the flow increases when more than one river connects to the estuary, in particular if the confluence is located within the limits of the tide. The model can include tributaries. Hydrodynamic and advection-dispersion processes within the tributaries are calculated using the same method as for the main channel and the confluence between the tributaries and the main channel is represented by pseudo three-dimensional cells.

\subsection{Architecture}

The architecture of the model is built around six modules interacting with each other via a number of subroutines and parameters (Figure 1).

A core program controls the Input-Output interface, the time variables, and co-ordinates the various modules.

The bathymetry module reads and interpolates the bathymetry from available section profiles. The local width of the channel and the local hydraulic radius are determined in each cell.

The hydrodynamics module calculates the water elevation, the velocity and the salinity. The hydrodynamics variables are used in the subsequent modules as they define the local shear stress, the dispersion coefficients and the volume of water.

The sediment modules compute the concentration of particles in suspension. This includes the erosion and deposition processes. Two types of sediment are distinguished: cohesive sediments (silt, clay) that tend to aggregate and non-cohesive sediment (sand, gravels) that are less inter-dependant. Cohesive and non-cohesive sediment transport is calculated in two submodules.

Contaminants such as radionuclides or heavy metals may interact with cohesive sediment through adsorption/desorption processes. The contaminant transport module calculates the concentration of contaminants in the dissolved, suspended sediment and bed sediment phases.

\subsection{Functionality}

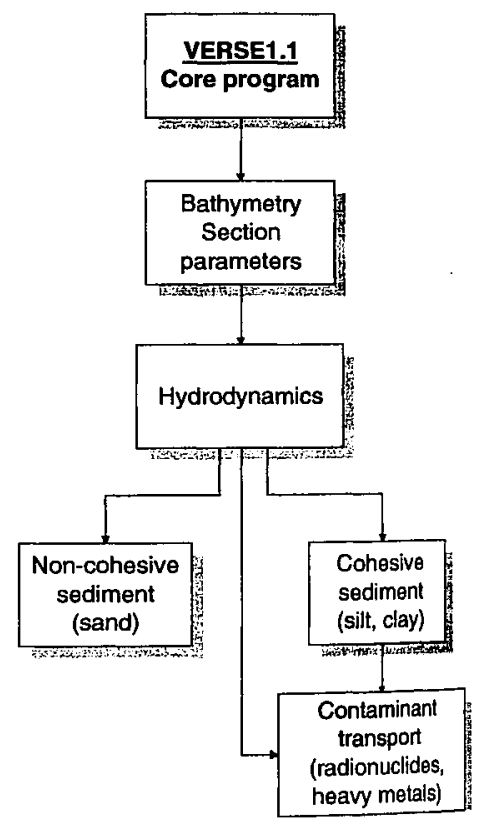

Figure 1. Model architecture

The hydrodynamics module predicts the tidal variation at the mouth of the estuary using the harmonic method. Up to 30 tidal constituents have been extracted from measurements of water elevation recorded over one month, after the influence of river discharge variations on the elevation have been subtracted. Shallow water distortion of the tidal curves has been included as a residual term that has been derived from a comparison between modelled and observed elevation.

The fresh water input into the main channel and tributaries can be interpolated from measured flow rates or set-up with pre-defined values for hypothetical scenarios. The high resolution of the vertical grid step together with limitations on the minimum depth of water, prevents the model from drying out under low river run-off and low water conditions.

Water elevation is determined from the global continuity equation for each section of the model. The horizontal component of the velocity is calculated from the momentum equation, whereas the vertical component of the velocity is determined from the local continuity equation. The dispersion coefficient is in part determined by saline stratification that can vary between flood and ebb tides. Stratification during the ebb tide traps sediment in the lower layers, nearer to the bed. This enhances its deposition and reduces the net seaward transport and is in part responsible for the tidal pumping mechanism ([1], [2]). 
Initial bed sediment distribution is specified from site observation. The transport of cohesive sediment takes into accounts the erosion and deposition processes. The erosion is proportional to the shear stress on the bed. A number of empirical formulas have been proposed from laboratory and field experiments ([3], [4], [5]). However, the erosion rate also depends on the consolidation of the deposit. Hayter [6] has proposed a multi-layer bed sediment model where each layer is characterised by a different degree of consolidation and therefore different erosion characteristics. A modification of this approach has been integrated into VERSE to define two broad categories of bed sediment ; an upper section represents the more mobile recently deposited sediment, and an underlying section presents harder, more consolidated sediment. The deposition of suspended sediment to the upper section is a function of local shear stress. The settling velocity of the particles depends on their relative density, but also on their flocculation stage. Mehta [7] derived an empirical relationship between the particle settling velocity and their concentration that has been used in the current model formulation. The erosion and deposition rate are calculated in each inundated cell of the model as each cell is in "contact" with the bed material either on the bottom or on the side of the channel. These processes are included in an advection-dispersion equation in order to calculate the SPM concentration at every time step.

The concentration of radionuclides is computed separately in the dissolved phase, the particulate phase and adsorbed onto bed sediment. The exchange of radionuclides between the three phases is represented uses a partition coefficient approach and can include instantaneous or non-instantaneous exchange rates.

The concentration of nuclides in each phase, from which the activity concentration is deduced, is calculated as follows:

$$
\begin{aligned}
& \frac{\partial C_{W}}{\partial t}=\underbrace{-u \nabla C_{w}}_{\text {advection }}+\underbrace{\frac{1}{b} \nabla \cdot\left(b K \nabla C_{w}\right)}_{\text {dispersion }}-\underbrace{Y C_{\text {SPM }} C_{W}}_{\text {adsorption }}+\underbrace{\frac{Y}{K_{d} C_{S P M} C_{P}}}_{\text {desorption }}-\underbrace{Y \rho_{b} \phi \frac{1_{s}}{R_{h}} C_{W}}_{\text {adsorption on deposit }}+\underbrace{\frac{Y \rho_{b} \phi l_{s}}{K_{d} R_{h}} C_{B}}_{\text {desoption fromdeposit }}-\underbrace{\lambda C_{W}}_{\text {decay }}+\underbrace{\lambda^{p} C_{W}^{p}}_{\text {parentdecay }} \\
& \frac{\partial C_{p}}{\partial t}=-\underbrace{u \nabla \nabla C_{p}}_{\text {advection }}+\underbrace{\frac{1}{b} \nabla \cdot\left(b K \nabla C_{w}\right)}_{\text {dispersion }}+\underbrace{Y \frac{C_{S P M}}{\rho} C_{W}}_{\text {adsorption }}-\underbrace{\frac{Y C_{S P M}}{K_{d} \rho} C_{P}}_{\text {desorption }}-\underbrace{\frac{w_{s}}{R_{h}}\left(1-\frac{\tau}{\tau_{d}}\right) C_{P}}_{\text {deposition }}+\underbrace{\frac{M_{e}}{\rho_{b} \phi l_{s}} C_{B}}_{\text {resuspension }}-\underbrace{\lambda C_{P}}_{\text {decay }}+\underbrace{\lambda^{p} C_{P}^{p}}_{\text {parentdecay }} \\
& \frac{\partial C_{B}}{\partial t}=\underbrace{\frac{\rho_{b} \phi 1_{s}}{\rho R_{h}} C_{W}}_{\text {adsorption ondeposit }}-\underbrace{\frac{Y \rho_{b} \phi l_{s}}{K_{d} \rho R_{h}} C_{B}}_{\text {desorption from deposit }}+\underbrace{\frac{W_{s}}{R_{h}}\left(1-\frac{\tau}{\tau_{d}}\right) C_{P}}_{\text {deposition }}-\underbrace{\frac{M_{e}}{\rho_{b} \phi l_{s}} C_{B}}_{\text {resuspension }}-\underbrace{\lambda C_{B}}_{\text {decay }}+\underbrace{\lambda C_{B}^{p}}_{\text {parentdecay }}
\end{aligned}
$$

The nuclide activity concentration in the dissolved phase, the particulate phase and the bed sediment are represented by $\mathrm{C}_{\mathrm{W}}\left(\mathrm{Bq} \mathrm{\textrm {m } ^ { - 3 }}\right), \mathrm{C}_{\mathrm{P}}\left(\mathrm{Bq} \mathrm{kg} \mathrm{kg}^{-1}\right)$ and $\mathrm{C}_{\mathrm{B}}\left(\mathrm{Bq} \mathrm{kg}^{-1}\right)$ respectively. The $\mathrm{SPM}$ concentration is defined by $\left.\mathrm{C}_{\mathrm{SPM}}\left(\mathrm{mg} \mathrm{l}^{-1}\right)\right)$. The other parameters represent:
u the velocity $\left(\mathrm{m} \mathrm{s}^{-1}\right)$;
b local width of the channel $(\mathrm{m})$;
$\mathbf{K}$
$\lambda$ and $\lambda^{p}$ the dispersion coefficients $\left(\mathrm{m}^{2} \mathrm{~s}^{-1}\right)$;
$\mathrm{Y}$ and $\mathrm{K}_{\mathrm{d}} \quad$ an adsorption rate $\left(\mathrm{m}^{3} \mathrm{~kg}^{-1} \mathrm{~s}^{-1}\right)$ and the partition coefficient $\left(\mathrm{m}^{3} \mathrm{~kg}^{-1}\right)$;
$\rho$
$\rho_{b}$ and $\phi \quad$ the bed density $\left(\mathrm{kg} \mathrm{m}^{-3}\right)$ and fraction of mud in the bed sediment respectively;
$\mathrm{R}_{\mathrm{h}}$
the hydraulic radius (m);
$\mathrm{W}_{\mathrm{s}} \quad$ the settling velocity of the suspended particles $\left(\mathrm{m} \mathrm{s}^{-1}\right)$;
$\tau$ and $\tau_{d} \quad$ the local shear stress $\left(\mathrm{m}^{2} \mathrm{~s}^{-1}\right)$ and critical shear stress for deposition $\left(\mathrm{m}^{2} \mathrm{~s}^{-1}\right)$;
$\mathrm{M}_{\mathrm{e}}$ the erosion rate $\left(\mathrm{kg} \mathrm{m}^{-2} \mathrm{~s}^{-1}\right)$. 
Radionuclide or other contaminant sources can be specified as local discharge point, diffuse source such as at the mouth or the head of the estuary, or in bed sediment.

Contamination of bed sediment is assumed to be limited to the upper layer of soft unconsolidated sediment. The unconsolidated sediment is divided into a number of layers of variable thickness. Depending on the erosion and deposition rates layers can be removed or added to the existing deposit. When erosion occurs, the flux of radionuclides transferred to the water column is calculated from the nuclide concentration in the eroded layers and the mass of sediment eroded. During sediment deposition the program updates the thickness and activity of the new sediment layer from the characteristics of the settling sediment. The model also allows direct diffuse exchange between radionuclides dissolved in the water of the overlying cell and sediment in the upper most layer.

Advection-dispersion and exchange between phases of a parent and up to nine daughter products can be calculated simultaneously. Distinct decay rates and partition coefficient ratios for the parent and each daughter can be included and is particularly important when decay chain products may provide a greater contribution to radiological dose, compared to the parent radionuclide that has been discharged.

\section{RESULTS}

\subsection{Validation}

The calibration and validation of the model have been based on field surveys in a macrotidal partially mixed estuary. Three instrument rig stations were deployed at the mouth, in the middle and at the head of the estuary. Each station included the following instrument arrays:

- a CTP-5 sensor that measured the local conductivity, temperature and pressure of the water, to provide values for salinity, temperature and water elevation;

- an ADCM current-meter recording the current velocity;

- an IR-40 infrared transmissometer measuring the level of turbidity and from which SPM concentration have been deduced.

Using mean daily river flow data, the model has been run over a four day period $(05 / 12 / 98$ to 09/12/98). The predictions of water elevation, current velocity and salinity have been compared with the
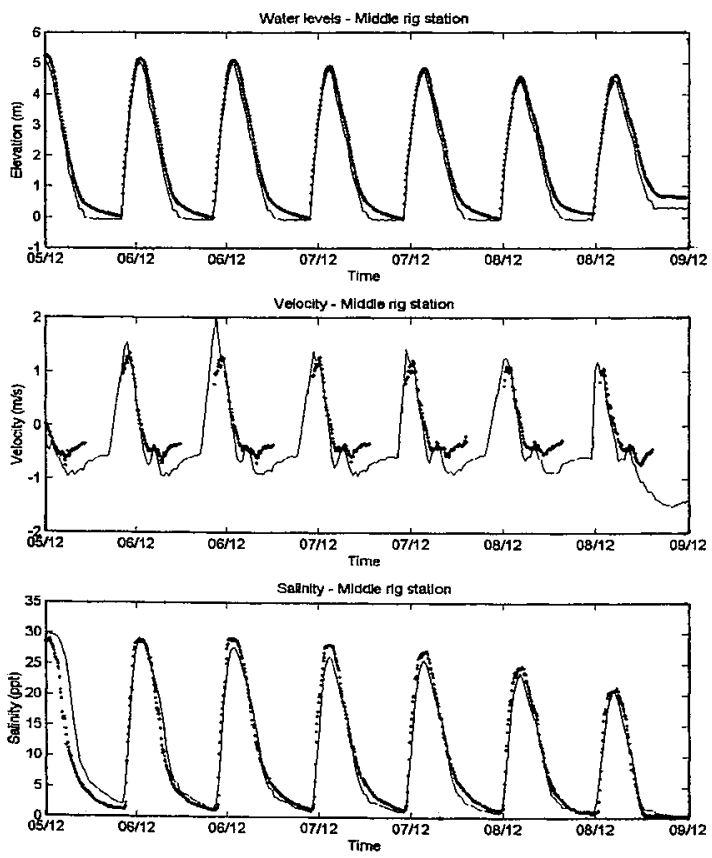

Figure 2. Model/data comparison for elevation, velocity and salinity in the middle part of the estuary. Data $=\cdot$ Model $=$ 
recorded values during this period. Figure 2 shows this comparison at the middle rig station. The model replicates changes in tidal elevation, flood velocity and salinity, but has a tendency to over predict ebb velocities.

Suspended sediment concentration is more difficult to predict as a large number of parameters and conditions can significantly influence the sediment transport predictions. The simulation run over a two day simulation period, from $05 / 12 / 98$ to $07 / 12 / 98$, shows that the model reproduces the variations in SPM concentration through a tidal cycle (Figure 3). However, the uncertainties in SPM concentration predictions remain significant and the predictions appear to be very sensitive to the initial bed distribution, the boundary conditions and parameters such as the critical shear stresses for erosion and

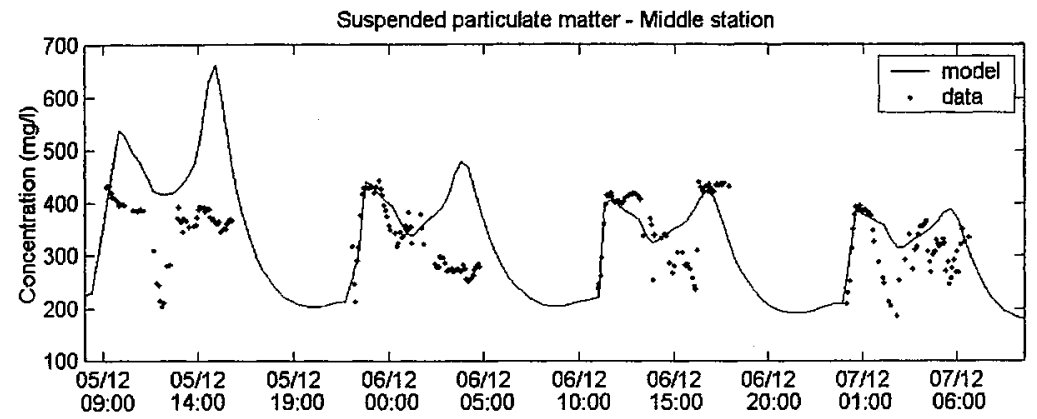

Figure 3. Model/data comparison for SPM concentration at the middle rig station

deposition, or the erodibility constants.

\subsection{Radionuclide dispersion}

As mentioned earlier, sources of contamination can be specified as a local discharge point, a diffuse source at the mouth or the head, as an initial bed sediment activity, or a combination of these sources. Figure 4 illustrates the dispersion of suspended sediment and associated contaminant through a tidal
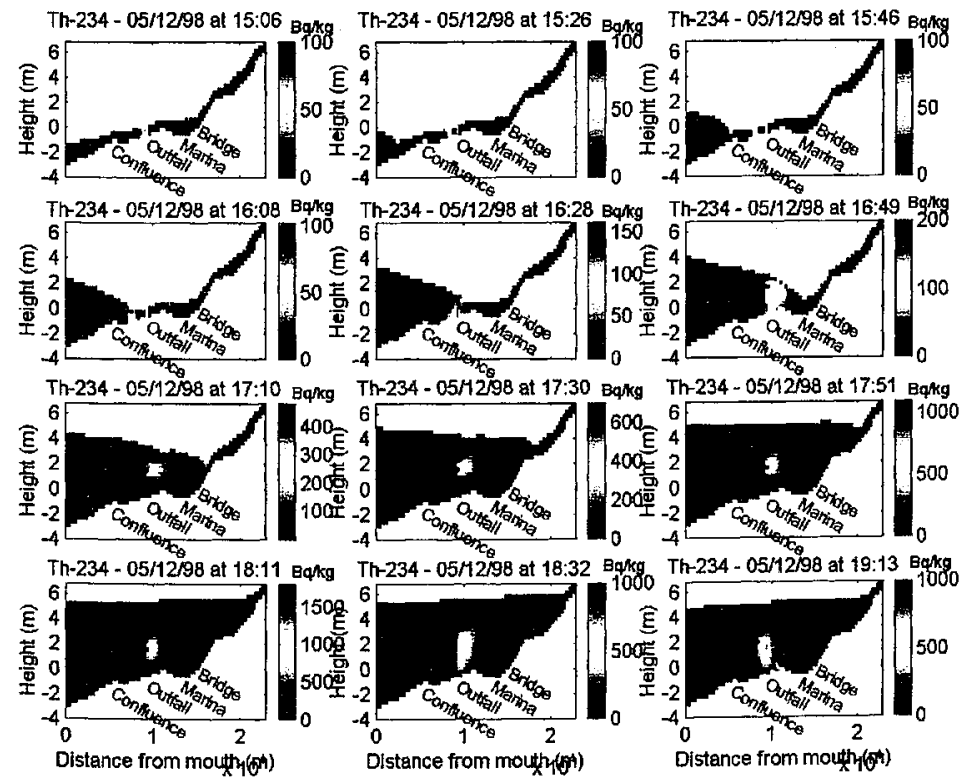

Figure 4 Effluent dispersion from low water to high water. 
cycle. The figure represents the side view of an estuary. In this simulation a particle reactive contaminant that is discharged in the dissolved phase rapidly partitions onto suspended sediment and is transpotted with the sediment upstream on the flood tide. The model shows that during spring tides and low river flow conditions suspended sediment and associated radionuclides can be transported and subsequently deposited in the upper reaches of the estuary.

The activity concentration of ${ }^{234} \mathrm{Th}$ in bed sediment is represented in Figure 5. The top graph of the figure shows the variations of the water elevation at the middle station and the vertical lines relate to the profiles shown below. The profiles show that, at this location, the radionuclide deposition to the sediment
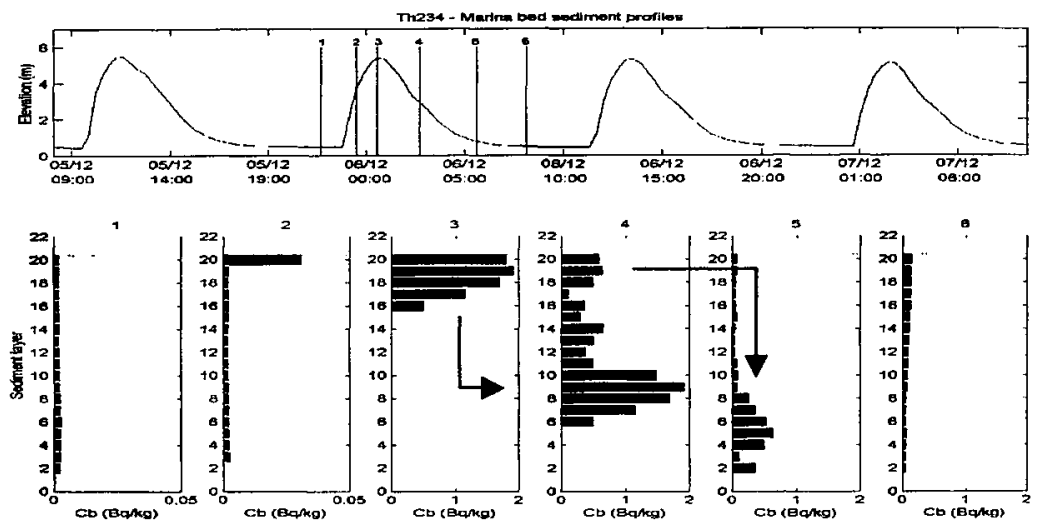

Figure 5. Activity profiles in bed sediment at the Marina location

occurs mainly between the end of flood and the beginning of ebb (periods 2 to 4 ). This is consistent with both the hydrodynamics conditions and the discharge location and periods. During ebb and low water, the current does not appear to be strong enough to erode the top layers. This preliminary result however must be considered more as an illustration of the model capabilities rather than as a description of the accumulation of contaminant in estuarine bed sediment.

\section{CONCLUSION}

A two-dimensional vertical numerical model has been developed in order to reproduce the hydrodynamics, the sediment transport and the contaminant dispersion in shallow, macrotidal partially mixed estuaries. The hydrodynamic predictions show a good agreement with data. The predictions of suspended sediment concentration reveal some discrepancies, although they reproduce reasonably well the observed turbidity variations. The sediment transport depends on a large number of uncertainties and empirical laws that make it more difficult to model and to predict.

VERSE provides an important tool for assessing the flux and fate of dissolved or particulate contaminants in estuarine environments, particularly those where decay or degradation products are important. The ability of the model to predict how changing tidal or river flow regimes will affect how bed contaminants are distributed through a sediment profile is essential for the prediction of radiological dose.

\section{References}

[1] Lyons M.G., Water, Air and Soil Pollution, 99 (1997), 141-148.

[2] Dyer K., Coastal and estuarine sediment dynamics (Wileys J. ed., 1990).

[3] Ariathurai R., Arulanandan K., Journal of the Hydraulics Division ASCE, 104 HY2 (1978), 279-283.

[4] Sheng Y.P., Lick W., Journal of Geophysical Research, Vol.84 C4 (1989), 1809-1826.

[5] Mehta A.J. et al., Estuarine Comparison (Kennedy V. ed., Academic press, 1982), 591-609.

[6] Hayter E.J., Estuarine Cohesive Sediment Dynamics (Mehta A.J. ed.), 326-359.

[7] Mehta A.J., Estuarine Comparison (Wiley ed., 1984), 290-325. 